Roxane Gagnon

Université de Genève

Kristine Balslev

Université de Genève

\section{Un geste pour comprendre la transformation des savoirs en formation et en classe : l'institutionnalisation}

\author{
Transformation of knowledge from teacher \\ training to initial vocational training
}

doi : 10.18162/fp.2016.299

\section{ésumé}

À la fois exemple de texte argumentatif oral dans le monde scolaire et situation déterminante pour les jeunes apprentis dans le monde professionnel,

l'entretien d'embauche constitue un objet de savoir biface. Le présent article pointe les tensions engendrées par cette double caractéristique et son effet sur la circulation des savoirs d'un contexte à un autre, grâce à des observations audio et vidéo de la mise en œuvre en formation et dans les classes de cet objet de savoir.

\section{Mots-clés}

formation des enseignants, entretien d'embauche, geste professionnel, institutionnalisation

Abstract

In vocational training, teachers frequently insert job interviews as a subject of instruction. In doing so, job interviews present two contrasted features: as an example of the argumentative text genre or as a crucial social situation. Through the analysis of video recordings of teacher training sessions about job interviews and of their actual teaching to apprentices, this paper shows the tensions that these two features generate and their effects on circulation of knowledge between teacher training and vocational training classrooms.

Keywords teacher education, job interview, professional act, institutionalisation
Dans un contexte où les enseignants sont encouragés à suivre des formations continues, voire contraints de le faire, la question de l'effet sur les pratiques enseignantes de ces formations est cruciale. Les contenus de savoir et démarche vus en formation sont-ils réinvestis dans la formation? Si oui, sous quelle forme? De quelle manière réapparaissent-ils dans les pratiques de classe des enseignants formés? Quels contenus sont relayés du contexte de la formation à celui de la classe? Et une fois que les contenus de la formation ont été testés en classe, quels contenus sont repris dans la suite de la formation? Telles sont les questions auxquelles tente de répondre le présent article.

Nous proposons d'approcher ces questions en étudiant la circulation des contenus de savoir d'un contexte à un autre et la construction de ces contenus par des acteurs endossant des rôles différents en fonction de ces contextes. Pour comprendre ce qui s'enseigne en argumentation orale dans le contexte de la formation continue et de l'école postobligatoire et pour cerner la manière dont ces contenus sont enseignés par les professionnels et la manière dont les professionnels s'approprient ces contenus, nous analysons les données selon un point de vue didactique, portant à la fois sur les savoirs et leurs transformations et sur l'activité même d'enseignement de ces savoirs. Nous faisons le choix de nous centrer sur un seul geste professionnel, l'institutionnalisation, car c'est par celui-ci que les contenus susceptibles d'être transférés dans un autre contexte sont mis en évidence. Nous observons d'abord sa matérialisation dans le discours des formateurs au cours de la formation continue. Ensuite, nous observons comment ce discours circule et se transforme en contexte de classe puis, par la suite, lors de l'entretien d'autoconfrontation à visée réflexive. 
Pour mener cette analyse, nous nous appuyons sur le corpus de données d'une recherche doctorale concernant l'enseignement de l'argumentation orale dans le postobligatoire (des adolescents âgés de 16 et 18 ans, en formation professionnelle), plus particulièrement sur les séances de formation et les leçons en classe de culture générale ayant trait à l'enseignement de l'entretien d'embauche (Gagnon, 2010). Dans la formation continue, comme dans tout autre cours portant sur un contenu spécifique, les moments d'institutionnalisation sont particulièrement intéressants parce qu'ils révèlent les contenus les plus significatifs. Ainsi, nos questions de recherche visent en premier lieu à un repérage de ces contenus dans la formation, puis dans le contexte de la classe de culture générale. Faisant l'hypothèse d'un effet de la forme sur le contenu enseigné, nous nous interrogeons également sur la modalisation de ces institutionnalisations dans les différents lieux. Nous cherchons ainsi à répondre aux questions suivantes :

- Quels contenus de savoir sont institutionnalisés dans la formation continue dispensée aux enseignants de culture générale?

- Comment se modalisent les discours rendant manifestes ces institutionnalisations dans les différents contextes?

- Quels contenus de savoir de l'objet entretien d'embauche sont négociés, repris et institutionnalisés dans la classe de culture générale?

Après un exposé du cadre théorique sont présentés les savoirs que l'entretien d'embauche permet de travailler ainsi que les savoirs utiles à son enseignement. Ensuite, les démarches méthodologiques et les analyses sont décrites. Nous émettons quelques éléments de prospective pour la formation en conclusion.

\section{Cadre théorique}

Geste didactique fondamental dans l'enseignement, l'institutionnalisation est notre objet d'étude, parce qu'il permet de mettre en relation les trois contextes et les trois rôles qu'endossent les acteurs (enseignant en formation; enseignant en exercice; enseignant en réflexion). Le premier contexte, celui de la formation continue, donne à voir le point de vue des enseignants formés à l'enseignement de l'argumentation orale. Le deuxième contexte, la classe de culture générale ${ }^{1}$ du CEPTA ${ }^{2}$, est le lieu où l'on enseigne un genre argumentatif oral, l'entretien d'embauche, à de jeunes apprentis mécaniciens; le point de vue est ici celui de l'enseignant en exercice. Le troisième contexte, celui de l'entretien d'autoconfrontation simple, donne à voir l'enseignante en réflexion sur sa pratique, le point de vue, plus introspectif, est guidé par les questions du formateur. Les modes d'interaction qui régissent les échanges entre les acteurs impliqués dans ces trois lieux portent des contenus de savoirs différents, liés aux contextes institutionnels. Nous postulons, avec Dugal et Léziart (2004), que les contenus de savoir circulent d'un contexte à un autre et que cette circulation transforme ces savoirs. 


\subsection{Circulation des savoirs dans les contextes de la formation}

La notion de circulation de savoirs postule l'existence de savoirs de natures différentes qui se combinent dans toute action de transmission et de communication (Dugal et Léziart, 2004). Il s'agit ici de cerner les savoirs professionnels déjà là et ceux en coconstruction des enseignants de culture générale à propos de l'enseignement de l'entretien d'embauche et de tracer leur circulation et leur transformation dans différents contextes. Par "savoirs professionnels ", nous entendons des savoirs relatifs à l'agir professionnel, issus des expériences en situation, des savoirs scientifiques appris, des prescriptions institutionnelles et du contact avec les pratiques sociales du terrain (Vanhulle, 2009b; Vanhulle, Balslev et Buysse, 2012). Les savoirs qui nous intéressent sont de différentes natures : les formateurs proposent des savoirs de référence issus de différents cadres à des enseignants de culture générale expérimentés, qui, enseignant déjà l'argumentation orale, ont constitué des savoirs et des pratiques liées à cet objet. La formation consiste alors davantage à amener les acteurs à saisir et à remettre en question leurs propres conceptions et à les confronter à des savoirs de référence (Vanhulle, 2008) qu'à leur apporter un savoir nouveau. Nous nous intéressons à la circulation entre différents lieux : celui de la classe de culture générale où se «voient » les pratiques enseignantes, celui de la formation où sont mis à disposition des savoirs de référence et négociés des savoirs à enseigner et des savoirs pour enseigner, et, enfin, celui de l'autoconfrontation où l'enseignante, dans une posture réflexive, met en discours sa pratique. La prise en compte de la circulation sous-tend que le lien qui va des savoirs de référence vers la pratique professionnelle n'est pas naturel, mais artificiel (Vanhulle, 2008). Ce lien se construit dans et par le discours.

Dans la perspective de la théorie de la transposition didactique (Chevallard, 1985/1991), ce croisement de lieux met en évidence les phénomènes de transposition interne et externe. Disposant du discours de l'enseignante sur ses pratiques, nous observons comment elle s'approprie les différents contenus de savoir de la formation continue. Ce discours ainsi que les interactions lors de la formation continue donnent une indication sur la manière dont l'objet de savoir est négocié et défini par l'enseignante. Les interactions dans la classe de culture générale permettent, quant à elles, de saisir comment le sens que les élèves attribuent aux objets d'enseignement participe à modifier cet objet. La transformation de l'objet dans ces différents cadres sera dépendante de sa didactisation, sa transposition, au moment de la formation.

Les données issues des trois cadres permettent d'appréhender la manière dont les savoirs didactiques (ces savoirs incluant les savoirs pour et à enseigner) et les savoirs issus de l'expérience circulent entre les différents lieux et de saisir leur articulation. Cette notion sous-tend que les savoirs ne sont pas des données fixes et met en question la distinction entre savoirs de la science et savoirs de terrain (Dugal et Léziart, 2004). Alors que la transposition didactique répond à une logique descendante ou ascendante, la circulation des savoirs s'inscrit dans une logique horizontale et permet dès lors d'intégrer le discours des acteurs (enseignants), d'accorder ainsi une place importante à la manière dont ils négocient, interrogent, interprètent et reformulent le savoir d'un contexte à un autre. 


\subsection{L'institutionnalisation : notre focale pour cerner la circulation des savoirs}

Pour Schneuwly (2000), le travail enseignant consiste en la transformation des modes de penser, de parler et d'agir des apprenants à l'aide d'outils sémiotiques. Aussi, toute situation d'enseignement ou de formation se construit sur une double sémiotisation : les objets passent par un processus de sémiotisation par «présentification" et par «pointage ». Le formateur ou l'enseignant transforme les objets en les rendant présents et en attirant l'attention sur l'une ou l'autre composante. Dans la formation étudiée ici, ces modes de penser, de dire et de faire se rapportent à des activités langagières complexes, telles qu'argumenter au cours d'un entretien d'embauche, mais aussi à ce qui touche l'activité d'enseignement de l'argumentation orale. L'analyse des pratiques effectives de la formation suppose l'adaptation d'outils habituellement consacrés à l'analyse des pratiques de classe au contexte de la formation et des interactions entre formateurs et enseignants formés.

Le geste d'institutionnalisation met en évidence les savoirs que l'enseignant juge centraux et permet à l'enseignant à la fois d'assurer à ses élèves que leur activité leur a fait retrouver des savoirs légitimes hors de l'institution et de fixer ces savoirs (Brousseau, 1998; Sensevy,2007). Étroitement lié à l'objet enseigné, il peut être qualifié de geste didactique (Aeby-Daghé et Dolz, 2008) et est porteur de significations.

Dans la formation continue des enseignants, le rôle des acteurs dans la légitimation des savoirs est primordial, les critères de reconnaissance de l'institutionnalisation doivent tenir compte des négociations entre formés et formateurs. Ajoutons que l'institutionnalisation peut être autant à la charge des formés qu'à celle du formateur. Cette conception de l'institutionnalisation est en accord avec l'idée que, dans la formation, les savoirs doivent faire l'objet d'une (re)problématisation pour que, d'une part, chacun des formés ait un rapport direct aux savoirs collectifs en train de se développer, et, d'autre part, pour contribuer à l'élaboration d'une vision du monde partagée (Martinand, 2006). Ainsi conçue, l'institutionnalisation tient compte de l'expertise des formés :

$\mathrm{Au}$-delà des régimes de médiations [...], il s'agit de penser ce quon pourrait appeler un régime d'expertise, où d'une part il faut recontextualiser, reformuler et reproblématiser des savoirs scientifiques vers d'autres pratiques, mais aussi problématiser et donc reconceptualiser des expériences pratiques ${ }^{3}$. (Martinand, 2006, p. 6)

\section{Choix méthodologiques pour cerner la circulation des savoirs}

Les institutionnalisations dans la formation et leur passage à d'autres lieux sont au cœur de notre étude, et nous présupposons que les savoirs institutionnalisés dans la classe proviennent partiellement de ce qui est institutionnalisé dans la formation. Afin de cerner les mécanismes discursifs sous-jacents à la circulation des contenus de savoir institutionnalisés et d'observer les dimensions de l'objet mises en avant par l'enseignant (Schneuwly et Dolz, 2009), notre démarche d'analyse se déploie en trois phases : 1) le repérage des institutionnalisations dans le discours; 2) l'analyse des contenus de savoir institutionnalisés; 3) l'étude des modalisations du discours dans les institutionnalisations. Il s'agit en définitive de saisir la construction des savoirs dans un double mouvement : transpositif, référant aux savoirs didactiques qui configurent l'objet, et circulationnel, lié aux significations de l'acteur. 
Le corpus de données analysé est constitué des transcriptions des journées de formation relatives à l'enseignement de l'entretien d'embauche dispensées au centre de formation professionnelle (correspondant à trois périodes de 60 minutes environ). Il comprend également la transcription de la séquence d'enseignement sur l'entretien d'embauche conduite par une enseignante formée en classe de culture générale que nous avons surnommée Rosalie. Cette séquence est constituée des transcriptions de quatre leçons de 45 minutes. Il inclut finalement l'entretien d'autoconfrontation simple dans lequel l'enseignante formée explicite sa pratique à la formatrice-chercheuse.

\subsection{Repérage des institutionnalisations}

Nos analyses ciblent les moments d'institutionnalisation et sont guidées par les questions suivantes : qu'est-ce qui est institutionnalisé? Comment? Qui participe à l'institutionnalisation?

Les moments d'institutionnalisation ont été repérés à l'aide de quatre critères (Schneuwly et Dolz, 2009):

1. une institutionnalisation s'adresse à un destinataire collectif (la classe ou le groupe des formés);

2. elle comporte une dimension de décontextualisation/recontextualisation qui marque le passage du savoir émergent à un savoir faisant partie de la classe, transférable à un contexte spatiotemporel autre que celui du hic et nunc;

3. elle comporte une généralisation marquée par des opérateurs linguistiques apparentés à la reprise (anaphore nominale ou pronominale, par exemple);

4. elle est marquée par la présence de marques de modalisation qui situent l'énoncé de base soit par rapport à la vérité, la nécessité, qui expriment des jugements d’ordre appréciatifs ou qui montrent des degrés d'adhésion du sujet de l'énonciation vis-à-vis de son énoncé.

Dans un deuxième temps, nous avons observé les contenus institutionnalisés repris dans la séquence de classe et au moment de l'entretien. De même, nous avons identifié le nombre d'occurrences de ces contenus dans les deux autres lieux (la classe de culture générale et l'entretien d'autoconfrontation).

\subsection{Contenus de savoir institutionnalisés}

L'entretien d'embauche est un genre argumentatif oral appartenant aux pratiques sociales de référence (Martinand, 1983), dont les contenus et la structure sont régulés à la fois par les conventions et par les différentes sphères de l'activité professionnelle. Son passage à la sphère scolaire nécessite la sélection et le pointage de contenus qui, bien qu'en adéquation avec la pratique sociale de référence, contribue à en faire un genre scolaire permettant, dans notre cas, le travail de capacités langagières orales.

L'entretien d'embauche est ici défini comme un échange dans lequel le candidat convoitant un emploi présente ses ressources de façon à ce qu'elles correspondent aux besoins de l'employeur potentiel et, idéalement, de façon à ce que celui-ci ou son représentant soit convaincu qu'il est le meilleur candidat possible. L'ensemble des savoirs à enseigner pour le genre textuel peut être regroupé en six composantes : la représentation de la situation de communication; la planification; la textualité; la voix; les ressources 
paraverbales; les supports écrits à l'entretien d'embauche (notes, curriculum vitae, listes de questions possibles, grilles d'évaluation, etc.).

La prédétermination des savoirs pour enseigner en lien avec l'entretien d'embauche s'organise en cinq regroupements: les finalités sociales et pédagogiques, les prescriptions institutionnelles, les connaissances des élèves, les méthodes d'enseignement et les travaux de recherche sur la formation enseignante (Hofstetter et Schneuwly, 2009).

L'institutionnalisation est liée à des choix stratégiques vis-à-vis de ces savoirs. L'analyse à priori des savoirs impliqués permet d'anticiper les choix des formateurs et des enseignants au regard de ce geste et d'identifier les institutionnalisations qui émergent des pratiques. Comme l'objet introduit dans la sphère de la formation est déjà connu des enseignants, certains ayant déjà enseigné le genre entretien d'embauche, il est problématisé par les enseignants en fonction de leurs connaissances et de leurs expériences. Quels savoirs sont jugés importants? Comment ces savoirs sont-ils pointés dans le cours de l'activité?

\subsection{Modalisations du discours}

À partir de l'étude des institutionnalisations dans la formation continue, nous avons analysé le cours de culture générale et l'autoconfrontation simple. Cette fois, deux questions orientent nos analyses : comment les dimensions institutionnalisées pendant la formation sont-elles activées par Rosalie et ses élèves dans le cours de culture générale? Comment sont-elles traitées par Rosalie lors de l'autoconfrontation? Il s'agit aussi d'observer comment l'enseignante modalise son discours. Pour répondre à ces questions, nous avons sélectionné quelques moments issus de la formation et les avons mis en lien avec des extraits de transcriptions d'interactions en classe et de l'entretien d'autoconfrontation.

Dans le cas de la formation à l'enseignement, l'appropriation des savoirs a en grande partie lieu par le biais des interactions verbales. Ainsi, le repérage du contenu et des modalisations du discours des acteurs offre une fenêtre ouverte sur cette appropriation : il permet de pointer ce que les acteurs disent, la manière dont ils imprègnent le discours et l'attitude qu'ils adoptent vis-à-vis de leurs propres énoncés (Charaudeau et Maingueneau, 2002). Nous retenons les catégories de modalisations du discours suivantes : les modalisations de type logique, consistant à positionner ses énoncés en fonction de lois générales, de vérités; les modalisations de type déontique, exprimant des valeurs, des opinions, des normes socialement légitimées, des références à l'ordre et au devoir; des modalisations de type pragmatique, qui portent sur les intentions des acteurs concernés dans telle ou telle situation sociale; et des modalisations appréciatives issues du monde de celui qui parle (Bronckart, 1996; Vanhulle, 2009a). Ajoutons que les énoncés ne sont pas toujours modalisés, dans ce cas-là on parle d'assertions. Lorsqu'ils sont modalisés, ils le sont à des degrés variables, allant d'un positionnement quasiment neutre à un positionnement fortement marqué. 


\section{Résultats : le passage des institutionnalisations d'un lieu à un autre}

Nous avons relevé dix moments d'institutionnalisation dans la séquence de formation et y avons identifié les contenus de savoir visés, l'initiateur et les participants à l'échange, trouvant ainsi réponse à notre première question de recherche : quels contenus de savoir sont institutionnalisés dans la formation continue dispensée aux enseignants de culture générale? Le classement de contenus de savoir a été effectué à partir des catégories de l'analyse à priori de l'objet de formation présentée dans la deuxième partie du présent texte.

Ces dix extraits ( $c f$. tableau 1, en annexe) montrent les contenus institutionnalisés au cours des interactions entre formateurs et enseignants en formation. Il s'agit, rappelons-le, des dimensions de l'objet jugées centrales, car elles ont fait l'objet d'une reformulation, problématisation par les formés, pour être pointées à nouveau par les formateurs.

\subsection{Les savoirs à et pour institutionnalisés dans les interactions en formation}

La représentation de la situation de communication figure parmi les savoirs à enseigner jugés centraux dans la formation. Nous décelons la volonté chez les enseignants de préparer les apprenants à l'entretien d'embauche, en étant le plus fidèles possible à ce qui se fait dans la sphère professionnelle. En arrièrefond, il semble aussi y avoir la crainte des enseignants d'apports universitaires décalés des besoins du marché du travail. C'est ce qui explique les négociations dans la formation au sujet de la plausibilité d'un entretien d'embauche qui impliquerait plusieurs candidats ou celles entourant le travail à partir des annonces. De plus, les enseignants en formation affirment vouloir préserver les apprenants des aspects négatifs liés à cette pratique sociale de référence; la logique de progression qui se dégage des échanges est que plus l'apprenant est en fin de formation, plus il est prêt à affronter un entretien d'embauche comparable à celui qui l'attendra à sa sortie de l'école.

Ce souci de réalisme explique également les négociations entourant la planification. Les discussions se rapportent au rôle et à la place de l'étayage argumentatif. Les enseignants s'intéressent aux questions à poser ou à ne pas poser, aux contenus et aux étapes de l'entretien d'embauche. D'autres stratégies argumentatives telles que la négociation ou la concession ne sont pas jugées centrales. Pour les enseignants, il ne semble pas important de repenser ou de mettre en question l'idée de travailler une stratégie argumentative plus entreprenante. D'ailleurs, dans la séquence de classe, il est question de «se soumettre » à l'intervieweur. Parmi les contenus liés à l'usage des ressources de la langue, seule la modalisation fait l'objet d'une institutionnalisation. Enfin, notons comme autre savoir à enseigner institutionnalisé, la part relative des ressources paraverbales (la communication non verbale). Autrement dit, pour eux, en faisant des élèves de meilleurs communicateurs, on les aide à pallier les « défauts » de leur apparence.

La question de la progression des contenus d'enseignement est prioritaire : les extraits 1, 4, 5, 7 se rapportent à l'idée de découper des contenus dans le temps. Au regard des méthodes, la question de la scolarisation du genre est, ici aussi, bien présente dans les préoccupations des enseignants. Il est question de la légitimité de l'étayage argumentatif comme contenu à enseigner. Quant aux différentes étapes de la séquence didactique présentée, on traite la production initiale et le travail de 
la documentation. Les connaissances des élèves et les implications pédagogiques constituent avec les démarches d'enseignement les principaux savoirs pour enseigner institutionnalisés. Notons le peu de place accordée à l'institution ainsi qu'aux savoirs sur les pratiques professionnelles.

\subsection{Du passage de la formation à la classe et à l'entretien... de nouveaux points de vue}

Nous avons procédé à un dénombrement des occurrences des contenus de savoir institutionnalisés dans la formation, une fois leur passage effectué de la formation à la classe et de la classe à l'entretien d'autoconfrontation simple.

Il ressort de ce repérage que les contenus institutionnalisés dans le contexte de la formation le sont aussi dans la classe. Ils sont repris avec des degrés d'insistance variables, la question de l'étayage des réponses à donner, des modalisations ainsi que la détermination de l'objet de la question reviennent le plus fréquemment. Ceci montre d'une part une grande cohérence entre les deux lieux et confirme d'autre part notre présupposé selon lequel ce qui est institutionnalisé lors de la formation préfigure l'institutionnalisation en classe. Pour ce qui est de l'entretien d'autoconfrontation, nos résultats sont beaucoup moins probants. Six des dix contenus institutionnalisés ne sont pas repris au cours de l'entretien et, au regard de la fréquence d'apparition des contenus, celle-ci est beaucoup plus faible que dans la séquence de classe. L'empan temporel plus court de l'entretien et le fait qu'il soit centré sur la seule pratique de l'enseignante expliquent en partie ces résultats. Par ailleurs, les savoirs pour enseigner sont prédominants dans les occurrences trouvées pour l'entretien.

En vue de montrer quelques reformulations et appropriations des savoirs dans les discours des acteurs en fonction des lieux, des extraits concernant quatre contenus de savoir institutionnalisés ont été choisis. Ils sont traités dans les extraits 2, 7 et 10 (voir tableau 1, en annexe). Les types de modalisations ont été mis entre crochets.

\section{Extrait 2}

Le contenu de savoir en jeu est pointé par une enseignante qui relate une lecture expliquant l'importance de l'apparence physique lors d'un entretien d'embauche.

E7 : moi, j'ai lu un petit article euh qui se trouve à notre centrale des doc qui dit ceci (il lit l'article) / nos études ont montré que $93 \%$ des impressions que vous produisez chez votre interlocuteur se jouent dès votre apparition même dès les premières secondes/ $55 \%$ sur votre apparence physique/ 38 \% par votre manière de parler et seulement $7 \%$ passe par ce que vous dites [assertion]

F1 : ah oui, tout à fait oui, c'est sûr que [logique]

E7 : donc, c'est moins de $7 \%$ sur le contenu

F1 : ouais, non c'est sûr, mais en même temps, on se dit, on se dit que :

E4 : dit n'importe quoi, mais raconte-le bien

E7 : il se fait une idée dès le (début?)

F1 : mais vous, vous êtes des enseignants de culture générale, donc vous vous dites : si on travaille 
l'entretien d'embauche, ce qu'on veut, ce qu'on veut, qu'est-ce qu'on veut est-ce qu'on veut juste les préparer à un entretien d'embauche ou on aimerait peut-être aussi (13'30) qu'ils apprennent peut-être à mieux communiquer [déontique]

La formatrice rompt la discussion à propos de l'importance de l'apparence physique en rappelant que le but est de préparer les apprentis à l'entretien d'embauche pour qu'ils apprennent à mieux communiquer. C'est ce savoir-là qui est institutionnalisé. L'extrait ci-après illustre comment il est reformulé en classe.

$\mathrm{E}$ : alors ça, de toute façon, c'est une règle absolue, n'est-ce pas? Ay et J.-D. on s'asseoit jamais avant de... d'y être invité bien sûr / [logique] est-ce que vous avez d'autres remarques euh peut-être sur la voix sur la posture

$[\ldots]$

$\mathrm{E}$ : effectivement ce qui effectivement généralement admis, c'est que on ne croise pas les mains comme ça (elle se croise les bras) parce que cette attitude-là peut être une attitude de défi ou une attitude de fermeture, ce qui, dans les deux cas, n'est pas positif donc on évite toujours d'avoir les bras croisés, ce que vous avez fait au début, mais après vous les avez décroisés [déontique]

Jo : moi, je m'en souviens pas

$\mathrm{E}$ : vous avez eu quelques minutes les bras croisés, même chose pour les jambes, hein alors l'attitude de fermeture absolue, c'est ça (elle le fait elle-même à sa place), les jambes croisées, les bras croisés, ça c'est vraiment le type qui veut pas se mouiller qui a une attitude de défi [déontique]

On voit que la question de l'apparence physique, rapidement évacuée lors de la formation continue, devient ici centrale. Quand le discours est modalisé, il l'est de manière logique ou déontique. De la formation à la classe, le savoir disparait : alors qu'il était question d'apprendre à mieux communiquer dans le premier lieu, la question de l'apparence physique prend le dessus dans le second lieu. Ceci s'explique par le souci des acteurs à tenir compte d'injonctions issues du monde professionnel et économique auquel seront confrontés les apprentis. Ce souci a pour effet d'annihiler l'un des objectifs de l'enseignement de l'entretien d'embauche, à savoir apprendre à mieux communiquer et argumenter. Cette institutionnalisation que l'on peut qualifier d'inconséquente illustre les tensions inhérentes à des situations scolaires proches de situations de la « vraie vie ».

\section{Extrait 7}

Dans cet extrait, la formatrice commence par rappeler l'importance pour les apprentis de tenir compte du contexte; ensuite une série de « règles » sont mentionnées, comme « ne pas critiquer une entreprise ou son ancien patron ».

F1 : parce que vous êtes, euh, vous, en plus, vous, vous l'enseignez avec des apprentis; moi, je suis avec vous un public très conciliant qui est en mesure de relativiser, de faire sa propre idée de ce que je peux, de tout ce que je peux dire, mais vous, vous êtes avec des jeunes apprentis qui veulent avoir des réponses, des solutions pis euh qu'est-ce que je dis, qu'est-ce que je dis pas, donc c'est d'essayer de leur apprendre aussi à tenir compte du contexte de la situation dans laquelle ils sont [pragmatique] est-ce que tu as le «feeling» (les doigts indiquent les guillemets) si on peut dire euh 
E9: mais c'est vrai qu'il y a des contradictions dans les ouvrages, mais il y a aussi des lignes, enfin, des choses qui se recoupent : ne pas critiquer l'entreprise, ne pas ne pas non plus critiquer son ancien patron, ne pas se dévaloriser, ça, c'est quand même des choses (brouhaha)

E3 : la base, quoi

F1 : pas d'agressivité

E9 : oui, pas d'agressivité

E4 : savoir esquisser des réponses par d'autres, par des questions, par d'autres questions

Dans la classe, ce savoir est traité majoritairement sous le mode logique ou déontique. Le savoir institutionnalisé en formation est quasiment repris tel quel dans la classe, de façon homothétique, comme l'illustre l'extrait suivant :

$\mathrm{E}$ : ça c'est quelque chose qu'il faut absolument éviter de faire NE JAMAIS CRITIQUER LA BOÎTE que vous venez de quitter parce que le le recruteur qu'est-ce qu'il va penser

él : ben qu'il va faire pareil avec eux

$\mathrm{E}$ : exactement $[\ldots]$ donc ça c'est une règle absolue : NI la boîte ni les collaborateurs avec qui on a travaillé, même s'ils sont... si vous avez une opinion déplorable sur eux, si ça s'est hyper mal passé et même si ces gens ont mauvaise réputation sur Genève. ON EN PARLE JAMAIS dans un entretien d'embauche si on vous dit, euh, est-ce que vous allez...H vous vous êtes bien entendus, vous noyez le poisson, vous vous débrouillez pour ne pas que : rentrer dans ce débat-là ni les produits ni la boîte ni l'organisation ni les gens qui travaillent

Lors de l'entretien d'autoconfrontation, l'enseignante reformule l'importance pour les apprentis de tenir compte du contexte. On peut y déceler une trace de l'appropriation d'un contenu de savoir institutionnalisé en formation.

$\mathrm{E}$ : oui ben je crois que je t'en ai déjà donné un exemple [logique], par exemple, euh, le niveau de langage, euh, il faut qu'ils s'habituent eux à passer d'un langage à l'autre dans leur vie quotidienne, on parle pas au patron comme on parle au copain, etc., juste ça, ça, on le fait dès l'école primaire en Suisse et parfois ça leur ça leur échappe [déontique]

\section{Extrait 10}

F1 : l'étayage, c'est comment on peut donner des exemples, justifier un fait, par exemple, êtes-vous, euh, êtes-vous ponctuel OUI // bon alors là, moi, si vous êtes dans mon équipe pis vous répondez oui, moi je dis non non non, c'est pas une bonne réponse oui parce que j'arrive toujours à l'heure [...] [déontique]

F1 : pis après ça c'est d'étayer pour montrer essayer de mousser sa candidature davantage [pragmatique] c'est euh c'est une forme de, c'est la forme d'argumentation qu'on peut travailler justement c'est l'étayage pis après (mouvement d'oscillation de la main), la négociation quand on travaille l'entretien d'embauche (...) [déontique] 
E8 : mais le but, c'est quand même de (mouvement de rotation de la main) pas tout argumenter parce que sinon on va perdre l'essentiel [pragmatique] / je pense qu'il y a des [logique]

F1 : dans l'arguH ce que je veux dire par là c'est vraiment juste de pas donner de réponse sans euh un oui ou un non, de pas répondre juste oui ou non (...) [appréciative]

E2 : étayer c'est aussi d'être authentique, de donner un peu de soi et puis // pas besoin de s'étaler non plus [...] [déontique]

F1 : mais si on se dit on travaille l'étayage aujourd'hui, alors là on va précisément demander justement de systématiquement étayer tout ce qui n'est pas, tout ce qui n'est pas xxx [déontique]

Le contenu de savoir que la formatrice et les enseignants institutionnalisent ensemble peut se résumer comme suit : argumenter signifie étayer, c'est-à-dire justifier. Toutefois, l'apport d'E2 « dévie » ce contenu de savoir, puisqu'elle ajoute qu' «étayer, c'est aussi être authentique " puis " pas besoin de s'étaler non plus... ». La formatrice reprend ceci, en affirmant que dans cette activité il s'agit justement de «systématiquement étayer ». Dans la classe, la question de l'étayage est reprise en fonction d'un cas précis, celui d'un élève se présentant à un entretien avec des dreadlocks.

$\mathrm{E}$ : bon alors au moment où (la recruteuse) demande à Bu s'il est capable de s'adapter, bon, elle fait référence à votre coiffure (l'élève a des dreadlocks) qu'il ait une coiffure jeune enfin : bon, et elle s'inquiète, en fait, sa question traduit une inquiétude sur votre capacité à vous intégrer dans une équipe de gens plus âgés que vous / c'est ça qu'elle traduit là [logique] donc il va falloir la rassurer, donc ce que vous avez essayé de faire, vous avez dit moi je pense ça me fait du bien d'être avec des... dans une ambiance de travail sérieuse, etc. euh vous auriez pu rajouter, euh, $\mathbf{j}$ 'ai un look jeune, mais je suis tout à fait adaptable, quelque chose comme ça, hein, il faut la rassurer parce que : elle exprime une inquiétude en vous disant est-ce que vous allez réussir à vous adapter // euh : / elle vous demande si vous avez / si vous êtes capable de travailler en équipe vous dites oui moi je peux travailler aussi bien seul qu'en groupe ce qui est bien hein [déontique]

Alors que dans la formation, l'activité argumentative est décrite comme une activité d'étayage et de justification, dans la classe, l'étayage est orienté vers un cas précis et concret : l'apparence physique et sa perception. Il est à noter que l'étayage argumentatif n'est pas abordé dans l'entretien d'autoconfrontation.

L'ensemble de ces extraits montre différents cas de circulation des contenus de savoir. Dans certains cas, le contenu est repris quasi tel quel d'un lieu à l'autre (extrait 7), dans un autre cas, le contenu institutionnalisé en formation est mis de côté pour laisser la place à un contenu qui avait été critiqué négativement par les formateurs (extrait 2); et, enfin, nous avons relevé un cas où le contenu est présenté de manière abstraite dans la formation et de manière plus contextualisée dans la classe (extrait 10).

\section{Conclusions et perspectives}

Nous avons fait le pari dans cette contribution d'analyser le passage des institutionnalisations dans trois contextes différents : la formation continue sur l'argumentation orale, la classe de culture générale et l'entretien d'autoconfrontation simple. En cours de route, l'analyse des interactions entre formateurs et enseignants nous a menées à redéfinir nos critères de repérage de l'institutionnalisation de manière à 
rendre compte des phénomènes de circulation des savoirs propres à la formation. Il s'avère que tous les contenus de savoir institutionnalisés en formation ont été repris dans la classe. À quelques exceptions près, ce qui est institutionnalisé lors de la formation annonce l'institutionnalisation en classe. Pour ce qui est de l'entretien d'autoconfrontation simple, nos analyses, moins concluantes, montrent une très faible reprise des contenus institutionnalisés en formation, ceux-ci n'intègrent que des savoirs pour enseigner. Comme Rosalie n'est pas habituée à ce type d'entretien et à se regarder enseigner, ses propos sont centrés sur les aspects qui lui paraissent les plus « évidents », en lien avec la conduite de classe.

Notre problématique et nos questions de recherche nous ont conduites à créer une démarche méthodologique pour repérer la circulation des savoirs. Dans un premier temps, nous avons sélectionné des extraits dans lesquels des contenus de savoir étaient institutionnalisés; puis nous avons cherché à « suivre » ces contenus dans les différents lieux par une analyse de contenu des interactions s'y déroulant. Cette démarche permet en effet de tracer les contenus de savoirs, néanmoins, elle force à revoir l'essence même d'un contenu de savoir. En effet, en considérant que chaque contenu est clairement défini et limité, il est quasi impossible de le «retrouver » ailleurs. Par conséquent, cette démarche oblige à considérer les contenus de savoirs dans leur contexte et à prendre en compte le caractère mouvant et situé de chacun des contenus.

En ce qui a trait aux dimensions de l'objet entretien d'embauche priorisées, nous avons constaté que les enseignants formés accordaient une grande importance à l'instauration de situations de communication réalistes dans la classe. Il y a à préparer l'élève à ce qu'il va affronter à sa sortie de l'école, mais en évitant le plus possible de le déconsidérer. Ces priorités se lient de près au contexte de la formation professionnelle, fortement en lien avec certains impératifs économiques et sociaux. Il y a aussi le fait que les élèves en formation professionnelle ont souvent connu un parcours scolaire cahoteux, ce qui amène parfois les enseignants à vouloir leur rendre la tâche moins exigeante. On veut préserver ces élèves, en prenant appui sur les productions des élèves et sur les échanges autour de celles-ci plutôt que d'exposer un savoir décontextualisé et réutilisable, visant le développement de savoirs langagiers. On retrouve ici l'une des tensions dans l'enseignement auprès des élèves en difficulté mise en évidence par Perrin-Glorian (2012) : donner la priorité à des problèmes complexes qui nécessitent une recherche ou favoriser l'engagement des élèves dans la résolution de problèmes qui tiennent compte des connaissances dont ils disposent.

La forme du discours varie d'un lieu à un autre. Si les modalisations déontiques et appréciatives apparaissent de manière plus fréquente dans la formation, le discours de l'enseignante en classe est marqué par une forte présence d'assertions, et de discours logique et déontique. Ce dernier constat confirme notre hypothèse d'un mode d'interaction plus symétrique entre formateurs et formés, lié au processus de circulation de savoirs. En revanche, la forme plus rigide et non discutable des contenus de savoirs liés à l'argumentation dans la classe a pour effet de transformer celui-ci en une règle à laquelle les élèves ont à se soumettre et non pas un savoir à questionner et discuter. L'enseignement de l'entretien d'embauche à des apprentis en dernière année de formation comporte de nombreux enjeux professionnels et personnels rendant impossible un travail sur l'argumentation détaché, dans lequel les savoirs ne sont pas recontextualisés en fonction des objectifs professionnels des élèves. Dans cette optique, il serait intéressant de comparer l'institutionnalisation de l'enseignement du genre argumentatif en se basant sur deux supports contrastés : une pratique sociale déterminante pour l'avenir professionnel des apprentis et une pratique détachée de cet avenir professionnel. 


\section{Références}

Aeby-Daghé, S. et Dolz, J. (2008). Des gestes didactiques fondateurs aux gestes spécifiques à l'enseignement-apprentissage du texte d'opinion. Dans D. Bucheton et O. Dezutter, Le développement des gestes professionnels dans l'enseignement du français: un défi pour la recherche (p. 83-105). Bruxelles : De Boeck.

Bronckart, J.-P. (1996). Activité langagière, textes et discours. Pour un interactionisme socio-discursif. Lausanne-Paris : Delachaux et Niestlé.

Brousseau, G. (1998). Théorie des situations didactiques. Grenoble : La Pensée Sauvage.

Charaudeau, P. et Maingueneau, D. (2002). Dictionnaire d'analyse du discours. Paris : Seuil.

Chevallard, Y. (1985/1991). La transposition didactique. Grenoble : La Pensée Sauvage.

Dugal, J.-P. et Léziart, Y. (2004). La circulation des savoirs entre recherche et formation : l'exemple des concepts didactiques lors d'une action de formation de conseillers pédagogiques. Revue française de pédagogie, 149(1), 37-47. http://dx.doi.org/10.3406/rfp.2004.3171

Gagnon, R. (2010). Former à enseigner l'argumentation orale : De l'objet de formation à l'objet enseigné en classe de culture générale (Thèse de doctorat, Université de Genève). Repéré à https://archive-ouverte.unige.ch/unige:6777

Hofstetter, R. et Schneuwly, B. (2009). Introduction. Savoirs en (trans)formation. Au cœur des professions de l'enseignement et de la formation. Dans R. Hofstetter et B. Schneuwly (dir.), Savoirs en (trans)formation: Au cour des professions de l'enseignement et de la formation (p. 7-40). Bruxelles : De Boeck. http://dx.doi.org/10.3917/dbu.hofst.2009.01.0007

Martinand, J.-L. (1983, juin). Cours. Questions pour la recherche : la référence et le possible dans les activités scientifiques scolaires. Atelier international d'été : Recherche en didactique de la Physique, La Londe les Maures - France.

Martinand, J.-L. (2006). Relations entre recherche et expertise dans un laboratoire de didactique curriculaire de sciences et techniques. Manuscrit d'une conférence « Entre pratique enseignante, formation et recherche : circulation des savoirs et reproblématisation ». Fès.

Perrin-Glorian, M.-J. (2012). Comment améliorer l'enseignement des mathématiques pour tous, y compris les élèves de milieu social défavorisé? Évolution d'un questionnement en didactique des mathématiques. Dans M.-L. Elalouf, A. Robert, A. Belhadjin et M.-F. Bishop (dir.), Les didactiques en question(s) (p. 100-110). Bruxelles : De Boeck.

Schneuwly, B. (2000). Les outils de l'enseignant - Un essai didactique. Repères, (22), 19-38. Repéré à http://ife.ens-lyon.fr/publications/edition-electronique/reperes/INRP RS022 2.pdf

Schneuwly, B. et Dolz, J. (2009). Des objets enseignés en classe de français : le travail de l’enseignant sur la rédaction de textes argumentatifs et sur la subordonnée relative. Rennes : Presses universitaires de Rennes.

Sensevy, G. (2007). Des catégories pour décrire et comprendre l'action didactique. Dans G. Sensevy et A. Mercier (dir.), Agir ensemble: L'action didactique conjointe du professeur et des élèves (p. 13-49). Rennes: Presses universitaires de Rennes.

Vanhulle, S. (2008). Au cœur de la didactique professionnelle, la subjectivation des savoirs. Dans Y. Lenoir et P. Pastré (dir.), Didactique professionnelle et didactiques disciplinaires en débats : un enjeu pour la professionnalisation des enseignants (p. 227-255). Toulouse : Octarès.

Vanhulle, S. (2009a). Des savoirs en jeu aux savoirs en «je». Cheminements réflexifs et subjectivation des savoirs chez des jeunes enseignants en formation. Berne/Neuchâtel : Peter Lang.

Vanhulle, S. (2009b). Savoirs professionnels et construction sociodiscursive de l'agir. Bulletin suisse de linguistique appliquée, (90), 167-188.

Vanhulle, S., Balslev, K. et Buysse, A. (2012). Comprendre les processus discursifs de la construction de savoirs professionnels et leurs effets régulateurs en termes de développement. Dans J. Clénet, P. Maubant et D. Poisson (dir.), Formations et professionnalisations : à l'épreuve de la complexité. (p. 117-150). Paris : L'Harmattan. 


\section{Notes de bas de page}

1 Bloc interdisciplinaire, l'enseignement de la culture générale est organisé par thèmes, ceux-ci s'articulent aux domaines d'apprentissage Langue et communication et Société.

2 L'acronyme signifie Centre d'enseignement professionnel technique et artisanal. La formation professionnelle en Suisse est sous la responsabilité de l'Office fédéral de la formation professionnelle et de la technologie (OFFT). Depuis 2010, le centre est dénommé CFP (Centre de formation professionnelle).

3 Les soulignements sont de l'auteur.

4 Les lettres renvoient aux formateurs F1 et F2 ainsi qu'aux neuf enseignants participant à la formation. Pour ne pas surcharger le tableau, nous avons retranscrits des extraits des moments d'interactions avec institutionnalisations, ce qui explique que certains participants à l'échange n'apparaissent pas. Les conventions de transcription sont les suivantes : / // /// : pauses de durée variable; demanH : unité inachevée; $\uparrow \downarrow$ : intonations montante et descendante; (entre parenthèses) : commentaires du transcripteur.

\section{Annexe}

\section{Tableau 1}

Moments d'institutionnalisation dans la séquence de formation

\begin{tabular}{|c|c|c|}
\hline $\begin{array}{l}\text { Contenus de savoir institution- } \\
\text { nalisés }\end{array}$ & Extraits de séquence & $\begin{array}{l}\text { Initiateur et } \\
\text { participants }\end{array}$ \\
\hline $\begin{array}{l}\text { 1) Savoir pour } \\
\text { Manière d'enseigner: } \\
\text { entretien d'embauche (ci-après } \\
\text { EE) en fonction du propre métier } \\
\text { des apprentis ou autre métier / } \\
\text { différences } 1^{\text {re }} \text { ou dernière année } \\
\text { d'apprentissage } \\
\text { Progression des } \\
\text { apprentissages : } \\
\text { Savoir à } \\
\text { Représentation de la situation } \\
\text { de communication de l'EE } \\
\end{array}$ & $\begin{array}{l}\text { F1 : il y a... il y a peut-être une façon de le faire en première qui est } \\
\text { différente de la façon de le faire en troisième, mais moi je } \\
\text { pense quili faut quand même plus, euh, plus ils vont être habiles, } \\
\text { meilleurs, ils vont être par rapport quand on parle de compétences } \\
\text { relationnelles et sociales, ça peut pas nécessairement être sur le } \\
\text { poste au début on va commencer avec un poste qui est autre } \\
\text { pis y aller plus sous forme de jeu. }\end{array}$ & $\begin{array}{l}\text { F1 } \\
\text { avec E9, E1, } \\
\text { F2, E5 }\end{array}$ \\
\hline $\begin{array}{l}\text { 2) Savoirà } \\
\text { impact de la communication } \\
\text { non verbale } \\
\text { Savoirs pour } \\
\text { Finalités sociales et } \\
\text { pédagogiques }\end{array}$ & $\begin{array}{l}\text { F1 : mais vous vous êtes des enseignants de culture générale, } \\
\text { donc vous vous dites si on travaille l'entretien d'embauche ce } \\
\text { qu'on veut, ce qu'on veut, qu'est-ce qu'on veut } \uparrow \text {, est-ce qu'on } \\
\text { veut juste les préparer à un entretien d'embauche ou on } \\
\text { aimerait peut-être aussi qu'ils apprennent peut-être à mieux } \\
\text { communiquer [... } \\
\text { parce que c'est tellement subjectif ces questions d'appa- } \\
\text { rence là, c'est... c'est je sais pas on peut pas } \\
\text { E1 : moi, je pense aussi quand on a travaillé sur, euh, pour mieux } \\
\text { communiquer dans ce genre de on améliore tout ce côté } \\
\text { global xxx concret donc c'est très utile quoi. } \uparrow\end{array}$ & $\begin{array}{l}\text { F1 avec } \\
\text { participation } \\
\text { de E1 }\end{array}$ \\
\hline
\end{tabular}




\begin{tabular}{|c|c|c|}
\hline $\begin{array}{l}\text { 3) Savoir à } \\
\text { Planification } \\
\text { Cerner l'objet de la question, } \\
\text { l'implicite, contenus sur lesquels } \\
\text { porte la question }\end{array}$ & $\begin{array}{l}\text { F1 : oui, fallacieuses parce que je pense qu'on a le droit de ne pas } \\
\text { répondre, mais faut juste savoir ne pas répondre, pis savoir } \\
\text { quand aussi ne pas répondre, mais encore là j'ai pas la science } \\
\text { infuse parce que je pense que ça// dépend toujours de la situation }\end{array}$ & F1 \\
\hline $\begin{array}{l}\text { 4) Savoir à } \\
\text { Représentation de la } \\
\text { situation de communication } \\
\text { Fidélité à la pratique sociale de } \\
\text { référence; les formes du débat } \\
\text { (entretien de groupe) } \\
\text { Savoir pour } \\
\text { Enseigner l'EE = faire maitriser } \\
\text { un genre }\end{array}$ & $\begin{array}{l}\text { F1: [... écoutez, moi je pense que ça peut être toujours dans l'idée } \\
\text { de travailler, on travaille donc nous on est à l'école, on essaie de } \\
\text { leur faire euh maitriser un genre [...] non, mais, travailler } \\
\text { l'entretien de groupe, comment par exemple est-ce que je peux, } \\
\text { euh, tenir compte de ce qui a été pour essayer après ça moi de me } \\
\text { présenter il y a une... il y a une complexité par en tout cas par } \\
\text { rapport à l'entretien en tête à tête, euh }\end{array}$ & F1 \\
\hline $\begin{array}{l}\text { 5) Savoirs pour } \\
\text { Le travail à faire sur la } \\
\text { documentation }\end{array}$ & $\begin{array}{l}\text { F1 : le premier travail qui peut être fait, c'est juste de voir en } \\
\text { lisant une annonce est-ce que je suis capable moi en tant } \\
\text { qu'apprenti de dégager les : besoins les attentes, donc c'est juste } \\
\text { de comprendre un texte qui est un texte d'annonce ensuite le } \\
\text { travail qui pourrait être fait ensH, après c'est de dégager ce } \\
\text { qui n'est pas dit dans l'annonce, mais qui pourrait aussi être } \\
\text { pertinent donc soit en allant faire une recherche sur Internet } \\
\text { ou en téléphonant ou même en allant s'entretenir avec des } \\
\text { gens ou en s'entretenant avec des gens qui font déjà le métier, } \\
\text { qui travaillent déjà là }\end{array}$ & F1 \\
\hline $\begin{array}{l}\text { 6) Savoir à } \\
\text { Planification : les questions } \\
\text { à poser } \\
\text { Délai de réponse }\end{array}$ & $\begin{array}{l}\mathrm{F} 1 \text { : ça dépend nous je crois qu'on en est venu à dire que ça dépend } \\
\text { toujours de bon [...] } \\
\mathrm{F} 1 \text { : ou il y a une façon peut-être polie, je vous remercie pour } \\
\text { l'entretien est-ce que je peux vous demander si : est-ce que je vais } \\
\text { avoir de vos nouvelles dans, dans, je sais pas } \\
\text { E7 : mais, ce qui importe, c'est la formulation }\end{array}$ & F1 avec E7 \\
\hline $\begin{array}{l}\text { 7) Savoirs pour } \\
\text { Les manières d'enseigner } \\
\text { Les réponses à donner aux } \\
\text { élèves, } \\
\text { les régularités du genre } \\
\text { Savoirs à } \\
\text { Planification } \\
\text { Structure et contenus de } \\
\text { l'entretien d'embauche }\end{array}$ & $\begin{array}{l}\text { E9: mais c'est vrai qu'il y a des contradictions dans les ouvrages, } \\
\text { mais il y a aussi des lignes enfin des choses qui se } \\
\text { recoupent : ne pas critiquer l'entreprise, ne pas ne pas non } \\
\text { plus critiquer son ancien patron, ne pas se dévaloriser, ça, } \\
\text { ''est quand même des choses [...] } \\
\text { E4 : savoir esquisser des réponses par d'autres, par des } \\
\text { questions, par d'autres questions [...] } \\
\text { F1: ben, vous avez une banque de réponses possibles à donner à } \\
\text { vos apprentis, ce sera à vous de voir laquelle vous laquelle vous } \\
\text { choisirez / }\end{array}$ & $\begin{array}{l}\text { E9 avec partici- } \\
\text { pation de E4 }\end{array}$ \\
\hline
\end{tabular}




\begin{tabular}{|c|c|c|}
\hline $\begin{array}{l}\text { 8) Savoir pour } \\
\text { Les manières d'enseigner } \\
\text { Exercice : faire argumenter les } \\
\text { élèves sans aucune préparation }\end{array}$ & $\begin{array}{l}\text { F1 : c'est sûr qu'avec des apprentis donc vous pouvez vous décidez } \\
\text { toujours en fonction de... de vos décisions vous pouvez soit les } \\
\text { faire travailler sur des... des postes en lien avec leur formation ou } \\
\text { quelque choseH } \\
\text { E9: mais ça, c'est intéressant comme exercice à faire en classe [...] } \\
\text { de leur demander d'argumenter sans aucune préparation } \\
\text { de leurs compétences euh :c'est bien }\end{array}$ & $\begin{array}{l}\text { F1 avec } \\
\text { participation } \\
\text { de E9 }\end{array}$ \\
\hline $\begin{array}{l}\text { 9) Savoir à } \\
\text { Textualisation (modalisation) }\end{array}$ & $\begin{array}{l}\text { F1 : ben, c'est-à-dire que c'est pour évoquer des exceptions sans } \\
\text { dévaloriser la candidature, c'est si jamais si on : se dit qu'on doit } \\
\text { dire la vérité, si jamais c'est sûr qu'il a, il devrait peut-être être } \\
\text { avant dans l'ordre, parce que c'est sûr qu'effectivement, ça, ça c'est } \\
\text { moins fort pour intéresser ou pour faire rire donc le seul retard que } \\
\text { je m'autoriserai sera pour mon enterrement, c'est un peu drôle } \\
\text { [...] } \\
\text { F1 : c'est toujours l'humour c'est ça peut être très efficace [déontique], } \\
\text { mais c'est toujours assez difficile [....], mais vous avez un } \\
\text { beau travail à faire sur la modalisation avec ces différentes } \\
\text { affirmations vous pouvez leur faire travailler justement cette } \\
\text { (mouvement de gauche à droite des mains) question de dire les } \\
\text { choses avec différentes modalités toujours et pis de les faire } \\
\text { aH après ça écouter eux à partir de des entretiens }\end{array}$ & $\mathrm{F} 1$ \\
\hline $\begin{array}{l}\text { 10) Savoir pour } \\
\text { Les manières d'enseigner } \\
\text { Comment/pourquoi travailler } \\
\text { l'étayage; } \\
\text { Donner des réponses avec } \\
\text { arguments } \\
\text { Savoir à } \\
\text { planification (étayage } \\
\text { argumentatif) }\end{array}$ & $\begin{array}{l}\text { F1 : l'étayage, c'est comment on peut donner des exemples, justifier } \\
\text { un fait par exemple [...] } \\
\text { F1: pis, après ça, c'est d'étayer pour montrer, essayer de mousser } \\
\text { sa candidature davantage, c'est, euh, c'est une forme de, c'est } \\
\text { la forme d'argumentation qu'on peut travailler justement c'est } \\
\text { l'étayage pis après la négociation quand on travaille l'entretien } \\
\text { d'embauche [...] } \\
\text { E8 : mais le but, c'est quand même de (mouvement de rotation de la } \\
\text { main) pas tout argumenter parce que sinon on va perdre l'essen- } \\
\text { tiel / je pense quili y a des [...] } \\
\text { E2 : étayer, c'est aussi de... de d'être authentique, de donner un peu } \\
\text { de soi et puis // pas besoin de s'étaler non plus [...] } \\
\text { F1: mais si on se dit on travaille l'étayage aujourd'hui, alors là on va } \\
\text { précisément demander justement de systématiquement étayer } \\
\text { tout ce qui n'est pas, tout ce qui n'est pas xxx }\end{array}$ & $\begin{array}{l}\text { F1 avec partici- } \\
\text { pation de E2 }\end{array}$ \\
\hline
\end{tabular}

\section{Pour citer cet article}

Gagnon, R. et Balslev, K. (2016). Un geste pour comprendre la transformation des savoirs en formation et en classe : l'institutionnalisation. Formation et profession, 24(3), 3-18. http://dx.doi.org/10.18162/fp.2016.299 